\title{
Broken-Bond Rule for the Surface Energies of Noble Metals
}

\author{
I. Galanakis, G. Bihlmayer, V. Bellini, N. Papanikolaou, R. Zeller, S. Blügel, $\uplus$ and P. H. Dederichs \\ Institut für Festkörperforschung, Forschungszentrum Jülich, D-52425 Jülich, Germany
}

(February 1, 2008)

\begin{abstract}
Using two different full-potential $a b$ initio techniques we introduce a simple, universal rule based on the number of broken first-neighbor bonds to determine the surface energies of the three noble metals $\mathrm{Cu}, \mathrm{Ag}$ and $\mathrm{Au}$. When a bond is broken, the rearrangement of the electronic charge for these metals does not lead to a change of the remaining bonds. Thus the energy needed to break a bond is independent of the surface orientation. This novel finding can lead to the development of simple models to describe the energetics of a surface like step and kink formation, crystal growth, alloy formation, equilibrium shape of mesoscopic crystallites and surface faceting.
\end{abstract}

PACS numbers: 71.15.Nc, 71.15.Cr, 71.20.Gj

The surface energy represents a fundamental material property. It is given by half the energy needed to cut a given crystal into two half crystals. As such the surface energy naturally depends on the strength of the bonding and on the orientation of the surface plane. A variety of experimental techniques have been developed to measure the surface energy [1], but all measurements are performed at high temperatures where surfaces are badly defined. The most comprehensive data stem from surface tension measurements in the liquid phase and by extrapolating the resulting orientation-averaged surface free energies to zero temperature [2,3]. The knowledge of the orientation-dependence of the surface energies is necessary to predict the equilibrium shape of a mesoscopic crystal and to study a series of important phenomena in materials science like crystal growth, creation of steps and kinks on surfaces, growth, stability and alloy formation of thin films or surface-melting faceting.

The lack of experimental data can be replaced by ab initio calculations. Due to the development of the density functional theory during the last two decades, ab initio methods are able to calculate many physical properties with unprecedented accuracy. Methfessel and collaborators [4] were the first to study the trends in surface energy, work function and relaxation for the whole series of bcc and fcc $4 d$ transition metals, using a full-potential (FP) version of the linear muffin-tin orbital (LMTO) method in conjunction with the local density approximation (LDA) to the exchange-correlation potential. In the same spirit Skriver and co-workers have used a LMTO technique to calculate the surface energy and the work function of most of the elemental metals [5]. Recently, Vitos and collaborators using their full-charge Green's function LMTO technique in the atomic sphere approximation (ASA) in conjunction with the generalized gradient approximation (GGA) elaborated a very useful database that contains the low-index surface energies for 60 metals in the periodic table [6. Also many semi-empirical [7] and tight-binding [8] studies exist.

In this contribution we show, using precise $a b$ initio techniques, that irrespective of the orientation the surface energies of $\mathrm{Cu}, \mathrm{Ag}$, and $\mathrm{Au}$ are simply proportional to the number of broken bonds between a surface atom and its nearest neighbors; for all surface orientations, except the (111) and (100), one has to take into account in the total number of broken bonds also the nearest bonds lost by the subsurface atoms. We demonstrate this in calculations for the low-index surfaces (111), (100), and (110) as well as for four vicinal surfaces. The resulting anisotropy ratios, i.e. the ratio of the surface energy for a given surface orientation with respect to the (111) surface energy, practically always agree with the "ideal" broken bond ratios, i.e. the number of broken bonds between nearest neighbors for this surface with respect to the (111) surface. This novel finding implies that (i) for the noble-metal surfaces the interaction between an atom and its second and further neighbors is very small and, that (ii) the charge rearrangement caused by the bond breaking does not significantly change the strength of the remaining bonds. Therefore, the energy needed to break a bond does not depend on the orientation, so that for each noble metal the surface energy for only one orientation is needed. We show that highly accurate calculations are compulsory to obtain these results.

To perform the calculations, we have used both the full-potential screened Korringa-Kohn-Rostoker (FKKR) method [9], and the full-potential linearized augmented plane wave (FLAPW) method [10] as implemented in the FLEUR code in conjunction with LDA. All calculations have been performed using the experimental lattice parameters: $3.61 \AA$ for $\mathrm{Cu}, 4.09 \AA$ for $\mathrm{Ag}$, and $4.08 \AA$ for $\mathrm{Au}$. For the FKKR an angular momentum cut-off of $\ell_{\max }=3$ for the wavefunctions and of $\ell_{\max }=6$ for the multipole expansion of the charge density and the potential has been used. To calculate the charge density, we integrated the Green's function along a contour on the complex energy plane, which extends from the bottom of the valence band up to the Fermi level [11]. Due to the smooth behavior of the Green's functions for complex energies, only few energy points are needed; 27 points have been used. A very large number of $\mathbf{k}_{\|}$points in the irreducible part of the two-dimensional Brillouin zone (2D-IBZ) has been used for the decisive complex energies close to the Fermi level ( $\sim 300$ points for the (111) and up to $\sim 800$ points for the (110) surface). The surface energy 
$\gamma$ for a $N$-layer slab embedded in semi-infinite vacuum is given by $\gamma=\left(E_{s}-N E_{b}\right) / 2$, where $E_{s}$ is the total energy of the slab and $E_{b}$ is the energy per atom in the bulk crystal; note that for fcc crystals $N$ is also the number of inequivalent atoms in the slab. To be consistent for all the cases we used as $E_{b}$ the energy per atom of the central layer of the slab. We have converged the number of metal layers so that the surface energies are converged within $0.01 \mathrm{eV}$; we used 12 layers for the (111), 14 for the (100), and 18 for the (110) surface. In FLAPW we calculated the surface energies from the total energies of two films of different thickness: for the (111) surface 13- and 11-layer films were used, for the (100) orientation 15 and 13 layers and for the (110) surfaces 19- and 17-layer films were calculated. A basis set of 80-90 augmented plane waves per atom and 425, 325, and $408 \mathbf{k}_{\|}$points were used in the 2D-IBZ of the (111), (100), and (110) surface, respectively.

In Table we collect the scalar-relativistically calculated surface energies within both FKKR and FLAPW and we compare them with the values obtained by the LMTO in Ref. [6]. For $\mathrm{Cu}$ and $\mathrm{Ag}$, the absolute values calculated with all three methods agree nicely. For Au both FKKR and LMTO predict that the surface energies are very close to the $\mathrm{Cu}$ values, whereas FLAPW predicts similar surface energies for $\mathrm{Au}$ and $\mathrm{Ag}$ in agreement with previous FLAPW results, $0.66 \mathrm{eV}$ for $\mathrm{Ag}(100)$ and $0.67 \mathrm{eV}$ for $\mathrm{Au}(100)$ [12]. A pseudopotential technique on the other hand shows the FKKR behavior: $\gamma=0.58 \mathrm{eV}$ for $\mathrm{Ag}(100)$ and $0.70 \mathrm{eV}$ for $\mathrm{Au}(100)$ [13].

As central result we present in Fig. 1 the anisotropy ratios. We remark that both FKKR and FLAPW calculations produce practically the same anisotropy ratios for all the noble metals, while previous LMTO [6] and FP-LMTO 【] calculations gave anisotropy ratios that deviate considerably from the present results, especially for Ag. Notably, both FKKR and FLAPW give results that are very close to $4 / 3$ for the $\gamma_{(100)} / \gamma_{(111)}$ ratio and close to $6 / 3$ for the $\gamma_{(110)} / \gamma_{(111)}$ ratio. These are exactly the ratios between the number of first-neighbor broken bonds for these surfaces. This finding can lead to two independent conclusions. Firstly, the broken bonds between a surface or a subsurface atom and its second and further neighbors have a negligible contribution to the surface energy. Secondly, the energy needed to break a bond is the same for any surface orientation. This is surprising, since one expects that breaking a bond in a surface leads to a rearrangement of the electronic charge resulting in a strengthening of the remaining bonds, so that one needs more energy to break them. But it seems that this bond strengthening, due to the reduction of neighbors, is negligible for the noble metals. To examine whether this finding also holds for the vicinal surfaces we used the FKKR method and calculated the surface energies and the anisotropy ratios for the next four more close-packed surfaces (see Table III). The number of layers used in the calculations is 21 for the (113), 30 for the (331) and the (210), and finally 32 for the (112) surface. For all these surfaces the anisotropy ratios are close to the ratios given by the broken first-neighbor bonds. Au shows slightly larger deviations from these ideal ratios compared to $\mathrm{Ag}$ and $\mathrm{Cu}$, which attends $3.6 \%$ in the case of the (210) surface. So the free energy $\gamma_{(h k l)}$ in $\mathrm{eV} /($ surface atom) needed to create any surface with a Miller index $(h k l)$ reduces just to the product of $\gamma_{(111)}$ and the ratio of the first-neighbor broken bonds, $N_{(h k l)}$, and $N_{(111)}=3$ :

$$
\gamma_{(h k l)}=\frac{N_{(h k l)}}{3} \gamma_{(111)} .
$$

$N_{(h k l)}$ can be easily obtained for any fcc surface [14]:

$$
N_{(h k l)}=\left\{\begin{array}{ll}
2 h+k & h, k, l \text { odd } \\
4 h+2 k & \text { otherwise }
\end{array} \quad h \geq k \geq l .\right.
$$

Given the disagreement of our anisotropy ratios with the published results of [4,6], we have performed extensive tests regarding the accuracy and consistency of our calculations. With the FKKR method we have performed calculations also for the somewhat smaller LDA lattice constants. Moreover, we have used both the full-potential KKR code as well as the KKR code with ASA potentials and full-charge density. While the absolute values of the surface energies change, the anisotropy ratios are extremely stable. With the FLAPW code we have performed additional calculations with the GGA and have estimated the effect of spin-orbit coupling for Au. Whereas the latter tends to increase the absolute values of the surface energies, GGA lowers the absolute values, but again we obtained anisotropy ratios in very good agreement with the broken-bond rule.

In the course of these checks we identified the most probable reason for the failure of previous calculations. In Fig. 2 we present the convergence of the surface energy of $\mathrm{Ag}$ with respect to the square root of the number of $\mathbf{k}_{\|}$-points used to perform integrations in the full first Brillouin zone, as for this system the difference between our present results and the ones in Refs. [1] and [6] is the largest. The (111) surface energy is very sensitive to the number of $\mathbf{k}_{\|}$-points, while this is not the case for the (100) surface; the (110) surface behaves similar to the (100) surface. This sensitivity might arise from a surface state centered at the $\bar{\Gamma}$ point, which all three noble metals possess and which requires a very dense $\mathbf{k}_{\|}$-grid to account for it. This is in-line with the observation that the largest deviations are 
obtained for Ag, for which this surface state is closest to the Fermi level. In the upper panel of Fig. 2 we show the ratio between the (100) and the (111) surface energies, which follows the oscillations of the $\gamma_{(111)}$ energy. In Refs. [4] and [6] the square root of the number of $\mathbf{k}_{\|}$-points in the full Brillouin zone used is about 9 and 13, respectively. For these numbers of $\mathbf{k}_{\|}$-points the FLAPW method gives a ratio which is between 1.15-1.20 very close to the ratio of 1.18 obtained by both LMTO methods. Thus the non-convergence of the surface energies with the number of $\mathbf{k}_{\|}$-points is the reason for the differences seen in Fig. 1.

In the following we discuss the range of validity and the limits of the broken-bond rule. Firstly, we note that we obtain similar anisotropy results for the anisotropy ratios of the $\mathrm{Rh}, \mathrm{Ir}, \mathrm{Pd}$, and Pt surfaces, although with slightly larger deviations (about $\pm 3-5 \%$ ) from the ideal ratios [15]. On the other hand, if we compare with another brokenbond system, i.e. the single vacancy, we find no agreement at all. In this case each of the 12 nearest neighbors of the vacancy looses one bond, so that according to the above rule the vacancy formation energy should be $12 / 3$ times the (111) surface energy. In reality, however, it is for all three noble metals more than a factor two smaller. Analogously we expect that the cohesive energy is by the same factor $12 / 3$ larger than the (111) surface energy. The values estimated in this way for the cohesive energies of $\mathrm{Cu}, \mathrm{Ag}$, and $\mathrm{Au}(2.70 \mathrm{eV}, 2.26 \mathrm{eV}$, and $2.49 \mathrm{eV}$, respectively) are about $20 \%-25 \%$ smaller than the experimental data $(3.51 \mathrm{eV}, 2.99 \mathrm{eV}$, and $3.56 \mathrm{eV}$, respectively) [16].

Finally we studied the effect of lattice relaxations on the calculated surface energies using the FLAPW and allowing the three first layers to relax. Although the calculated surface energies change, the effect on the anisotropy ratios is much smaller. For $\mathrm{Cu}$, relaxations reduce $\gamma$ by $0.8 \%, 0.7 \%$ and $2.6 \%$ for the (111), (100) and (110) surfaces, respectively. The $(100) /(111)$ anisotropy ratio stayed unchanged by the relaxations while the relaxed $(110) /(111)$ ratio was 1.98 compared to the value of 2.01 for the unrelaxed structure. For Ag, the surface energy was reduced by $0.1 \%, 0.7 \%$ and $1.8 \%$ for the three low-index surfaces, respectively. The new anisotropy ratios are 1.27 and 1.93 slightly smaller than the original values of 1.28 and 1.95. Finally for $\mathrm{Au}$, relaxations reduce the surface energies by $0.2 \%$ and $0.8 \%$ for the (111) and (100) surfaces and the anisotropy ratio becomes 1.35 slightly smaller than the value, 1.37, for the unrelaxed structure. The $\mathrm{Au}(110)$ surface shows a large relaxation; the distance between the first and the second layer $\left(\Delta d_{12}\right)$ is reduced by $13.8 \%$, the $\Delta d_{23}$ is expanded by $6.9 \%$ and finally the $\Delta d_{34}$ is also reduced by $3.2 \%$. The surface energy is reduced by $6.5 \%$ and the anisotropy ratio is now 1.89 compared to the 2.04 for the unrelaxed structure, but remains close to the broken-bond rule value of 2.0. So even large relaxations have a rather small impact on the calculated anisotropy ratios which are reasonably described by the broken-bond rule.

Several experiments at high temperatures have been carried out mainly on gold crystallites 17 to determine the $\gamma$-anisotropy, but their interpretation is difficult. Entropy terms, describing the lower vibrational frequencies of the atoms at the surface as compared to the bulk, the formation of kinks and finally the creation of holes and pillboxes at the low-index surfaces, have to be added to the total free energy. Also at such high temperatures the surface-melting faceting [18], i.e. the break-down of a vicinal surface in a dry and a melted one, plays a predominant role. Recently, Bonzel and Edmundts [19] have shown that analyzing the equilibrium shape of crystallites at various temperatures by scanning tunneling microscopy can yield absolute values of the surface and step energies versus temperature, but this technique has not been yet applied. Also after the growth of a nanocrystal, there is a dynamical procedure towards its equilibrium, which can be modeled using the anisotropy of the surface energies at $0 \mathrm{~K}$ [20]. Similar problems arise from high-temperature measurements on single Au surfaces [21]. The easy calculation of the surface energies for the noble metals as a product of the $\gamma_{(111)}$ and the number of first neighbor broken bonds can be used to develop more complicated models describing the above phenomena. There are also other important applications in materials science like the growth and stability of a thin film or the growth of a crystal surface where the knowledge of the absolute values and the anisotropy of the surface energies is crucial.

In this Letter we have shown for different surface orientations that the surface energies of the noble metals accurately scale with the number of the broken nearest-neighbor bonds, so that the calculated anisotropy ratios always agree well with the ideal broken-bond ratios. We have demonstrated this in FKKR and FLAPW calculations for seven low-index and vicinal surfaces of $\mathrm{Cu}, \mathrm{Ag}$ and $\mathrm{Au}$. We believe that the simplicity of these results are of great interest for a variety of problems in materials science like step, kink, and alloy formation, crystal growth, surface-melting faceting or the shape of small crystallites on a catalyst.

Authors wish to thank H.P. Bonzel for helpful comments on the manuscript. Authors gratefully acknowledge support from the Psi-k TMR network (Contract No: FMRX-CT-0178), the TMR network of Interface Magnetism (Contract No: ERBFMRXCT96-0089) and the RT Network of Computational Magnetoelectronics (Contract No: RTN1-1999-00145) of the European Commission. 
+ Permanent address: Fachbereich Physik, Universität Osnabrück, D-49069 Osnabrück, Germany.

[1] V. K. Kumikov and Kh. B. Khokonov, J. Appl. Phys. 54, 1346 (1983).

[2] W. R. Tyson and W. A. Miller, Surf. Sci. 62, 267 (1977).

[3] F. R. Boer et al., Cohesion in Metals, North-Holland, Amsterdam, (1988).

[4] M. Methfessel, D. Hennig, and M. Scheffler, Phys. Rev. B 46, 4816 (1992).

[5] H. L. Skriver and N. M. Rosengaard, Phys. Rev. B 46, 7157 (1992); J. Kollár, L. Vitos, and H. L. Skriver, Phys. Rev. B 49, 11288 (1994); M. Alden, H. L. Skriver, S. Mirbt, and B. Johansson, Phys. Rev. Lett. 69, 2296 (1992).

[6] L. Vitos, A. V. Ruban, H. L. Skriver, and J. Kollár, Surf. Sci. 411, 186 (1998).

[7] S. M. Foiles, M. I. Baskes, and M. S. Daw, Phys. Rev. B 33, 7983 (1986); M. I. Baskes, Phys. Rev. B 46,2727 (1992); A. M. Rodríguez, G. Bozzolo, and J. Ferrante, Surf. Sci. 289, 100 (1993).

[8] M. M. Mehl and D. Papaconstantopoulos, Phys. Rev. B 544519 (1996); C. Barreteau, D. Spanjaard, and M. C. Desjonquères, Surf. Sci. 433-435, 751 (1999).

[9] R. Zeller et al., Phys. Rev. B 52, 8807 (1995); R. Zeller, Phys. Rev. B 55, 9400 (1997); K. Wildberger, R. Zeller, and P. H. Dederichs, Phys. Rev. B 55, 10074 (1997).

[10] E. Wimmer, H. Krakauer, M. Weinert, and A. J. Freeman, Phys. Rev. B 24, 864 (1981); M. Weinert, E. Wimmer, and A. J. Freeman, Phys. Rev. B 26, 4571 (1982).

[11] R. Zeller, J. Deutz, and P. H. Dederichs, Sol. State Commun. 44, 993 (1982); K. Wildberger, P. Lang, R. Zeller, and P. H. Dederichs, Phys. Rev. B 52, 11052 (1995).

[12] H. Erschbaumer, A. J. Freeman, C. L. Fu, and R. Podloucky, Surf. Sci. 243, 317 (1991); R. Eibler et al., Surf. Sci. 280, 398 (1993).

[13] N. Takeuchi, C. T. Chan, and K. M. Ho, Phys. Rev. B 43, 14363 (1991); ibid., 13899 (1991).

[14] J. K. Mackenzie, A. J. W. Moore, and J. F. Nicholas, J. Phys. Chem. Solids 23, 185 (1962).

[15] I. Galanakis et al. (unpublished).

[16] C. Kittel, Introduction to Solid State Physics, 1953.

[17] B. E. Sundquist, Acta Met. 12, 67 (1964); W. L. Winterbottom and N. A. Gjostein, Act. Met. 14, 1041 (1966); J. C. Heyraud and J. J. Métois, Acta Met. 28, 1789 (1980); Z. Wang and P. Wynblatt, Surf. Sci. 398, 259 (1998).

[18] G. Bilalbegivić, F. Ercolessi, and E. Tosatti, Surf. Sci. 280, 335 (1993); H. M. van Pixteren and J. W. M. Frenken, Europhys. Lett. 21, 43 (1993); H. M. van Pixteren, B. Pluis, and J. W. M. Frenken, Phys. Rev. B 49, 13 798 (1994).

[19] H. P. Bonzel and A. Edmundts, Phys. Rev. Lett. 84, 5804 (2000).

[20] M. Ozdemir and A. Zangwill, Phys. Rev. B 45, 3718 (1992); N. Combe, P. Jensen, and A. Pimpinelli, Phys. Rev. Lett. 85, $110(2000)$.

[21] U. Breuer and H. P. Bonzel, Surf. Sci. 273, 219 (1992).

TABLE I. Scalar-relativistic surface energies for the three noble metals using both FKKR and FLAPW compared with previous LMTO results from Ref. [6]. All results are given in $\mathrm{eV} /($ surface atom).

\begin{tabular}{|c|c|c|c|c|c|c|c|c|c|}
\hline \multirow[b]{2}{*}{$\gamma(\mathrm{eV}) \mid$} & \multicolumn{3}{|c|}{$\mathrm{Cu}$} & \multicolumn{3}{|c|}{$\mathrm{Ag}$} & \multicolumn{3}{|c|}{$\mathrm{Au}$} \\
\hline & FKKR & FLAPW & Lмто & FKKR & FLAPW & Lмто & FKKR & FLAPW & LMTO \\
\hline (111) & 0.67 & 0.62 & \begin{tabular}{l|}
0.71 \\
\end{tabular} & 0.57 & 0.51 & 0.55 & 0.62 & 0.50 & $\overline{0.61}$ \\
\hline (100) & 0.87 & 0.81 & 0.91 & 0.73 & 0.65 & 0.65 & 0.84 & 0.68 & 0.90 \\
\hline (110) & 1.33 & 1.25 & 1.32 & 1.11 & 1.00 & 0.95 & 1.28 & 1.01 & 1.32 \\
\hline
\end{tabular}

TABLE II. FKKR scalar-relativistic surface energies given in eV/(surface atom) for the seven more close packed surfaces together with the anisotropy ratios in parenthesis. $d$ is the distance between two successive layers and $a$ the lattice constant. $\mathrm{BB}$ is the number of first-neighbor broken bonds.

\begin{tabular}{|c|c|c|c|c|c|}
\hline & $\mathrm{Cu}$ & $\mathrm{Ag}$ & $\mathrm{Au}$ & $d / a$ & $\mathrm{BB}$ \\
\hline$\overline{(111)}$ & 0.675 & 0.566 & 0.623 & 0.5774 & 3 \\
\hline$(100)$ & (1.29) 0.874 & (1.29) 0.728 & (1.35) 0.842 & 0.5000 & 4 \\
\hline (110) & (1.97) 1.327 & (1.97) 1.113 & (2.06) 1.284 & 0.3535 & 6 \\
\hline (311) & (2.32) 1.564 & (2.31) 1.309 & (2.36) 1.468 & 0.3015 & 7 \\
\hline$(331)$ & (2.99) 2.016 & (2.97) 1.680 & (3.05) 1.900 & 0.2294 & 9 \\
\hline$(210)$ & (3.32) 2.240 & (3.29) 1.864 & $(3.45) 2.149$ & 0.2236 & 10 \\
\hline (211) & (3.34) 2.255 & (3.32) 1.877 & (3.39) 2.110 & 0.2041 & 10 \\
\hline
\end{tabular}




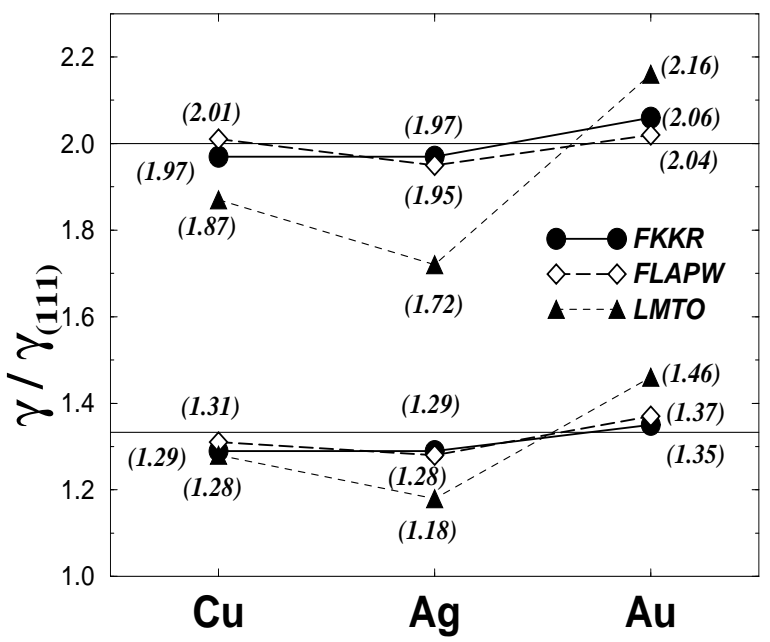

FIG. 1. The anisotropy ratios for the three noble metals, $\gamma_{(100)} / \gamma_{(111)}$ and $\gamma_{(110)} / \gamma_{(111)}$, using both FKKR and FLAPW. The LMTO results are from Ref. [6]. FP-LMTO calculations [4] for Ag produced similar results to those in Ref. [6]. Surface energies are calculated in $\mathrm{eV} /$ (surface atom). The two straight solid lines represent the ideal first neighbor broken-bond ratios; $4 / 3$ for $(100)$ and $6 / 3=2$ for $(110)$.
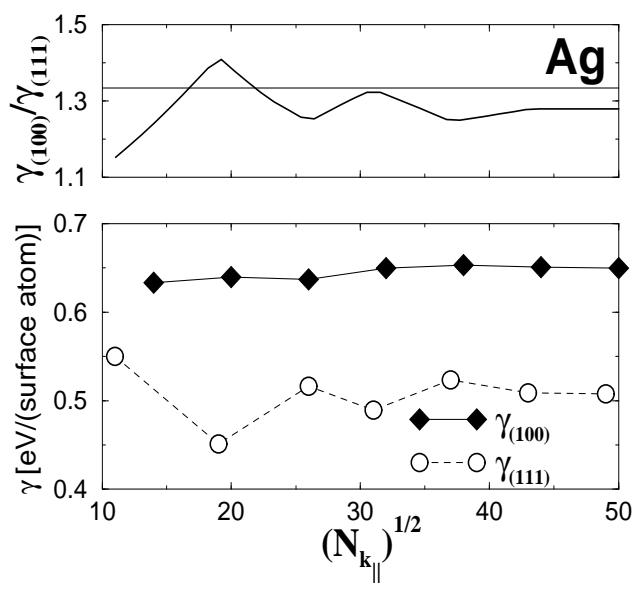

FIG. 2. Convergence of the surface energies $\gamma(111)$ and $\gamma(100)$ with th e square root of the number of $\mathbf{k}_{\|}$-points in the full first 2D Brillouin zone using the FLAPW method. The upper panel shows the resulting anisotropy ratio $\gamma_{(100)} / \gamma_{(111)}$ together with the ideal value of $4 / 3$. 